Revista Punto Género № 8. Diciembre de 2017

ISSN 0719-0417 / 103 - 114

\title{
Mujeres y estratificación social: un caso de sexismo intelectual*
}

Joan Acker. Traducción Laura Duclos

Re-impreso de Joan Acker "Women and Social Stratification: A Case of Intellectual Sexism" American Journal of Sociology 78 (Enero, 1973): 936-945, con autorización del editor.

Texto traducido al castellano por Laura Duclos, con la autorización de University of Chicago Press.

Fecha de recepción: 14 de enero 2018

Fecha de aprobación: 21 de enero 2018

\section{Presentación}

Publicado en 1973, este artículo ha sido pionero en el ámbito de los estudios de estratificación social, particularmente en el mundo anglosajón. La discusión metodológica instalada desde los años 1950 con los estudios de Blau y Duncan, y parte de la discusión teórica del ámbito clásico de la estratificación y la movilidad social, aún no se había estrellado contra los estudios feministas. Las dos tradiciones marxistas y weberianas, clásicas en los estudios de estratificación, tendían a considerar que respecto de la clase social, un atributo de la familia más que del individuo, bastaba en términos de medición con una evaluación de los atributos del principal sostenedor del hogar, en general el hombre. Simplificaba el acercamiento empírico a la noción de clase, a la vez que hacía sentido en sociedades como la norteamericana, donde la mayoría de las mujeres era dueña de casa. Sin embargo, varias y profundas evoluciones sociales en los años 1950 y 1960 ya no permitían sostener ni teórica ni empíricamente la forma en que los estudios de estratificación social se hacían - o más bien no se hacían - cargo de la posición de las mujeres. La resistencia del medio frente a este cambio necesario lleva a Joan Acker, académica de la Universidad de Oregon, a publicar este breve pero contundente artículo en un número especial del American Journal of Sociology, donde desarma meticulosamente cada una de las afirmaciones de la visión "convencional", que invisibiliza la posición de las mujeres, al subsumirla a la del varón del cual dependerían. Posteriormente, este artículo recibirá varias respuestas, y dará lugar a una amplia y fecunda controversia académica, que durará hasta los años 1980. Fallecida recientemente en 2016, Joan Acker ha sido una de las más contundentes expositoras de la teoría de género en los estudios de estratificación social, razón por la cual quisimos rendirle un homenaje al publicar este artículo en castellano, siendo la primera vez que este texto es traducido a nuestro idioma. Fue traducido en el marco de una asignatura de pregrado sobre estratificación social dictada por Emmanuelle Barozet en la carrera de sociología de la Universidad de Chile, por una de las estudiantes, Laura Duclos. Por ello, es un agrado ponerlo a disposición del público castellano parlante.

* Revisión de un artículo leído en el congreso anual de la Southwestern Sociological Association (1971), Dallas, Texas. 


\section{Mujeres y estratificación social: un caso de sexismo intelectual}

En los últimos diez años, los estudios empíricos y las discusiones especulativas sobre el desventajado estatus de las mujeres han aumentado rápidamente. A pesar de que la desigualdad social es el tema de los estudios de estratificación, solo una pequeña parte de este trabajo sobre la posición de las mujeres ha sido hecho por sociólogos y sociólogas en el campo de la estratificación social ${ }^{1}$. En efecto, el sexo rara vez ha sido analizado como un factor en procesos de estratificación y estructuras ${ }^{2}$, a pesar de ser probablemente uno de los criterios más obvios de diferenciación social y una de las bases más obvias de las desigualdades económicas, políticas y sociales. Muy pocos sociólogos han siquiera reconocido que hemos construido, exceptuando los estudios de la familia, una sociología que tiende a lidiar sólo con la mitad masculina de la humanidad.

La inclusión de la mitad femenina de la humanidad y del sexo como una dimensión central en el estudio de la sociedad daría lugar a una imagen más certera de la estructura social y a un mejor entendimiento de los procesos. Sin embargo, una consideración seria sobre el sexo como un factor social central requerirá una re conceptualización en muchas áreas de la sociología. El asunto central de este artículo son los problemas conceptuales y de método que surgen en el campo de la estratificación social cuando se asume que las mujeres son participantes significativas en la sociedad. Discuto, primero, los supuestos en

\footnotetext{
${ }^{1}$ Sociólogos de otras áreas han hecho una serie de contribuciones recientes que son relevantes para problemas de estratificación. Por ejemplo, Epstein (1970) discute la prominencia del estatus sexual en las carreras profesionales de mujeres en las ocupaciones más altas. Oppenheimer (1968) examina las bases de las etiquetas sexuales de los trabajos en la fuerza de trabajo americana. Wilensky (1968) analiza las relaciones entre la posición de las mujeres, el crecimiento económico y la ideología democrática. Etzioni (1969) investiga las relaciones entre estatussexual y la ocupación en las semi-profesiones. Algunos anteriores, pero aislados análisis, fueron Hacker (1951) y Myrdal (1944, apéndice 5), quienes discutieron acerca de las mujeres en cuanto grupo minoritario; Hughes (1949), quien coloca a las mujeres de carrera no tradicional en el rol de "hombres marginales"; Ellis (1962), quien estudia las correlaciones de movilidad en las mujeres profesionales; y Caplow (1954), quien dedica un capítulo a las mujeres en el mundo del trabajo. Han habido otras discusiones sociológicas sobre la posición de las mujeres (Komarovsky, 1950, 1953; Bernard, 1966, 1968), pero estos análisis no han sido integrados en los estudios de estratificación social.

${ }^{2}$ Lenski (1966) es uno de los pocos quienes reconocieron este problema en el campo de la estratificación. En Poder y privilegio, declara lo siguiente: "otro aspecto muy descuidado por el análisis de la distribución en las sociedades modernas es el sistema de clases basado en el sexo." También observa que: "en los análisis de las sociedades industriales avanzadas, es imposible ignorar o dar por obvios el rol del sexo en los procesos de distribución." Aun así, incluso Lenski hace un análisis poco profundo. El autor concluye su breve discusión de la posición de las mujeres (mencionada en 13 páginas de un total de 446 páginas en el texto) con la cómoda idea de que "para la vasta mayoría de las mujeres, la batalla por la igualdad sexual ha sido ganada." Podría haber cierta contradicción entre las declaraciones de que la batalla por la igualdad de sexo ha sido ganada y que el sexo sigue siendo un factor importante en el proceso distributivo. No obstante, este libro ha sido publicado antes de los movimientos de liberación de la mujer, en 1966, cuando tales inconsistencias, aunque bastante frecuentes, eran relativamente invisibles. Esta contradicción refleja las dificultades de la teoría de estratificación al tratar con el estatus de mujer.
} 
la literatura sobre la estratificación social acerca de la posición de las mujeres; segundo, algunos problemas de re conceptualización; $y$, tercero, algunas contribuciones para la comprensión de la sociedad, que pueden resultar del estudio de las mujeres en el sistema de estratificación.

\section{Supuestos sobre mujeres y estratificación}

En la literatura sobre estratificación, existen seis supuestos, a veces explícitos y a veces implícitos, sobre la posición social de las mujeres. Están enunciados más claramente por los funcionalistas, pero también se encuentran presentes en los trabajos de los nofuncionalistas y de los marxistas. ${ }^{3}$ Estos supuestos son:

1. La familia es la unidad en el sistema de estratificación.

2. La posición social de la familia está determinada por el estatus del hombre jefe de hogar.

3. Las mujeres viven en familias; por lo tanto, su estatus está determinado por el de los hombres a quienes están unidas.

4. El estatus de la mujer es igual al de su cónyuge, al menos en términos de posición en la estructura de clases, porque la familia es una unidad de evaluación equivalente (Watson y Barth, 1964).

5. Las mujeres determinan su propio estatus sólo cuando no están vinculadas con un hombre.

6. Las mujeres no son iguales a los hombres de muchas formas, y son diferencialmente evaluadas en base al sexo, pero esto es irrelevante para la estructura de los sistemas de estratificación social.

El primer supuesto, que la familia es la unidad en estratificación, es la base de los otros cinco. Estos supuestos, juntos, descartan con esmero la necesidad de considerar la posición de las mujeres en los estudios de estratificación social o de considerar la prominencia del sexo como una dimensión de estratificación ${ }^{4}$. Para ponerlo de otra forma, el destino de la mujer en el sistema de clase es determinado por el destino del hombre. Por lo tanto, es necesario estudiar sólo a los hombres.

3 Véase, por ejemplo, dos estudios recientes sobre la estructura de clase en Polonia y Checoslovaquia (Machonin, 1970; Wesolowski y Slomczynski, 1968), los cuales expresan de manera explícita los supuestos aquí delineados.

${ }^{4}$ Lenski (1966) hace la misma observación: "Esta negligencia [hacia las mujeres] se debe en gran medida a la tendencia de los sociólogos de tratar las familias, por sobre los individuos, como la unidad básica de los sistemas de estratificación" (402). 
¿Qué tan adecuados son estos supuestos? Aquí hay, creo, deficiencias de lógica y validez, las cuales discutiré brevemente.

1. La familia es la unidad en el sistema de estratificación social. -La elección de la familia como unidad puede basarse en la creencia de que todas las personas viven en familias. Obviamente esto no es verdad, dado que el $11 \%$ de la población mayor de 18 años es categorizada como individuos independientes en los datos de $1970 .^{5}$ Este supuesto también descansa en la validez de los otros cinco, los cuales examinaré en los próximos párrafos.

2. La posición social de la familia está determinada por el estatus del hombre jefe de hogar. - Esta es una cuestión investigable que ha sido poco investigada. En su lugar, investigaciones empíricas a menudo presuponen una respuesta a esta pregunta en su elección de indicadores de clase o posición social. Así, si el ingreso familiar es elegido como un indicador, implica que el ingreso familiar total determina su posicionamiento. Si la posición de clase es medida sólo por la ocupación o como un índice que incluye la ocupación, aquella del hombre jefe de hogar es invariablemente utilizada, asumiendo que su posición determina la posición de la familia. ${ }^{6}$

Hay una situación en la que el segundo supuesto es claramente inválido. La posición de la familia no puede ser determinada por el hombre jefe de hogar si no hay un hombre a la cabeza del hogar. Esta es la situación en una proporción significativa de familias estadounidenses. En base al censo de 1960, Watson y Barth (1964) estimaron que aproximadamente dos quintos de los hogares en Estados Unidos no tienen un hombre a la cabeza, en el sentido que supone el modelo tradicional de una pequeña familia nuclear. Encontraron que dos quintos de los hogares eran ya sea "mujeres o mujer jefa de hogar, o familias matrimoniales donde el marido está jubilado o si no, fuera de la fuerza laboral, desempleado, o sólo trabajando a tiempo parcial" (1964).

3. Las mujeres viven en familia; por lo tanto, su estatus está determinado por el de los hombres a quienes están unidas.- Este supuesto puede ser desafiado sobre la base de que no todas las mujeres viven en familias. Es más, el supuesto de que el estatus de la mujer está determinado por el del hombre al cual está unida, implica que las mujeres no poseen recursos de estatus propios. En una sociedad en la cual las mujeres, al igual que los hombres, poseen recursos de educación, ocupación e ingresos, evidentemente no es verdad que las mujeres no tengan bases para determinar su propio estatus. Si las mujeres tienen tales recursos, ¿por qué asumimos que no operan si la mujer está casada? Es

${ }^{5}$ Calculados a partir de las tablas 6, 8, y 44, Statical Abstract of the United States, 1971 (U.S., Oficina del Censo 1971, 36)

${ }^{6}$ Hofstetter (1970) explora este problema. Concluye que el auto-posicionamiento de clase de estudiantes universitarios encuestados podría estar determinado por los recursos combinados del padre y la madre, más que por aquellos sólo del padre. 
inconsistente jerarquizar a una mujer no-casada basándose en su educación y ocupación, sosteniendo, después, que estos factores no son de importancia para su estatus social y posición de clase al día siguiente de haberse casado ${ }^{7}$. Sin embargo, tal alteración abrupta del criterio de posicionamiento de clase en el momento de un cambio de estatus marital es necesario si queremos aceptar el supuesto que sólo las mujeres sin hombres determinan su propio estatus.

4. El estatus de la mujer es igual al de su cónyuge.-Una vez que cuestionamos el supuesto de que el estatus de la mujer está determinado por el hombre, debemos cuestionar también el supuesto de que el estatus de la mujer es igual al de su conyugue. Desde luego, esposa y esposo pueden ser iguales, pero no se puede seguir asumiendo una evaluación equivalente.

Incluso si todas las mujeres no tuviesen recursos independientes de creación de estatus, la igualdad de su estatus con el de sus maridos seguiría en cuestión. Se puede evaluar la igualdad en base a numerosas dimensiones. El prestigio en la comunidad, el estilo de vida, privilegios, oportunidades, asociación con grupos sociales, ingresos, educación, ocupación y poder podrían ser considerados en la evaluación de la igualdad entre esposo y esposa en la estructura de clases. La ocupación, equiparada por los funcionalistas con un rol social funcionalmente importante de tiempo completo, es a menudo utilizada como indicador de la posición de los hombres. No obstante, la ocupación de tiempo completo de muchas mujeres, la de la madre-dueña de casa nunca es considerada como un criterio de clasificación en estudios de estratificación. ¿Debemos concluir que este rol no es funcionalmente importante o no es una actividad de tiempo completo?, ¿o concluimos que sólo aquellas actividades que son directamente retribuidas financieramente pueden conferir estatus a los individuos o a las familias? Quizás sea esta otra pregunta que puede ser explorada mediante investigación empírica. Algo de evidencia investigativa hay para sugerir que las amas de casa cuyos maridos trabajan en una ocupación dada, tienen menos prestigio que las mujeres que, por sí mismas, están empleadas en la misma ocupación (Haavio-Mannila, 1969). No obstante, la evidencia para sostener o refutar el supuesto de igual estatus respecto a la estructura de clase es, desafortunadamente, escasa.

5. Las mujeres determinan su propio estatus sólo cuando no están unidas a un hombre. Este supuesto puede ser interpretado como una manera de hacer frente al hecho inconveniente de que algunas mujeres no están casadas o viviendo en el hogar de un familiar hombre.

6. Las mujeres se encuentran en una situación de desventaja en las jerarquías de riqueza, poder y prestigio, pero este hecho es irrelevante para el estudio de los sistemas de estratificación social. - Este supuesto está implícito en la literatura sobre estratificación.

\footnotetext{
${ }^{7}$ Muchas de estas observaciones también fueron hechas por Watson y Barth en una crítica aguda a algunos supuestos en la teoría de la estratificación y en una investigación publicada en 1964.
} 
Por una parte, saco esta conclusión de la escasa atención a la situación de las mujeres en la literatura sobre estratificación, y, por otra parte, de la existencia de una amplia evidencia de que las mujeres están excluidas de las posiciones de poder más altas, de que ganan menos que los hombres y de que están presentes en muy pequeñas proporciones en las ocupaciones más prestigiosas.

Pero, tal vez, la posición de las mujeres es irrelevante en la estructura del sistema en general. No lo creo. Por ejemplo, los hogares encabezados por mujeres representan casi el $40 \%$ de los que se encuentran bajo la línea de la pobreza (Ferriss, 1970). Estas estadísticas sugieren que las desventajas económicas y sociales asociados con ser mujer pueden tener impacto en las diferencias de clase en la estructura familiar. Cuando los teóricos de la estratificación hablan de algunas clases, en gran medida están hablando sobre mujeres. Es posible que algunas de las diferencias que discuten sean relativas al sexo más que a diferencias de clases. Quizás estas diferencias, por ejemplo, tengan efecto en los patrones de movilidad y la permeabilidad de los límites de clase, lo cual afecta al sistema más amplio de maneras más complejas.

En suma, no es adecuado ni útil asumir que las mujeres no tienen un rol relevante en los procesos de estratificación, independientemente de sus roles familiares y de sus lazos con hombres particulares. Si esta conclusión es razonable, se sugiere reconsiderar el estatus del sexo y la estratificación.

Como un primer paso en tal reconsideración, hago las siguientes suposiciones:

1. El sexo es una característica adscrita duradera, la cual (a) tiene efecto sobre la evaluación de las personas y las posiciones y (b) es la base de la persistente división sexual del trabajo y de las desigualdades basadas en el sexo.

2. La dicotomía sexual atraviesa todas las clases y estratos. (Esto también es válido para la etnicidad y la raza).

\section{Modelos de estratificación y sexo}

Una serie de problemas conceptuales surge cuando el sexo es considerado como una variable relevante de estratificación. Uno de esos problemas es el siguiente: ¿pueden las desigualdades basadas en el sexo ser integradas a un modelo conceptual de sistemas de estratificación? La mirada tradicional de las clases como agregados que ocupan posiciones similares en relación con los medios de producción, o posiciones similares en una o más jerarquías de riqueza, poder o prestigio, han hecho difícil lidiar con desigualdades que atraviesan las líneas de clase. Es más fácil asumir, como lo señalan Watson y Barth (1964), que la familia es una unidad, que los miembros de la familia son igualmente evaluados, y que, por lo tanto, no es importante investigar el estatus de las mujeres. 
Algunos desarrollos recientes en el estudio de la estratificación social quizás hagan más fácil considerar seriamente las desigualdades de sexo. Por ejemplo, existe una tendencia a expandir los estudios de estratificación para incluir una amplia variedad de desigualdades sociales estructuradas (Heller, 1969). De manera similar, existe una tendencia a alejarse de la preocupación exclusiva por las definiciones clásicas de clase y a estar preocupados por el individuo como unidad. A pesar de que esta tendencia empezó hace años atrás, ahora parece establecerse con bendiciones incluso en la persona de Parsons. Una recensión reciente señala que: "él [Parsons] también plantea objeciones serias a la relevancia de las definiciones clásicas de clase en el análisis de sociedades modernas, argumentando... que la unidad de estratificación de clase basada en la familia ya no puede seguir siendo útil nada más que como un complejo masculino de membresías colectivas adscriptivas y logradas, incluyendo su membresía en la organización" (Lauman, 1970).

Utilizando al individuo más que a la familia como unidad, quizás sea posible integrar el sexo en los modelos de sistemas de estratificación social al menos de dos maneras: (1) como una dimensión en estratificación que corte a través de las líneas de clase y produzca dos jerarquías interrelacionadas de posiciones o personas, o (2) como una base de evaluación que afecte las posiciones individuales en jerarquías particulares.

Una solución alternativa al problema de la integración en los modelos conceptuales de sistemas de estratificación de las desigualdades basadas en el sexo, podría no requerir abandonar las definiciones clásicas de clase. Las mujeres pueden ser consideradas como constituyentes de una agrupación tipo casta dentro de las clases sociales ${ }^{8}$. Las castas femeninas, usando esta aproximación, quizás tengan ciertos intereses y patrones de vida comunes. Adicionalmente, pueden compartir ciertas discapacidades e inequidades. Al mismo tiempo, las castas femeninas están incrustadas en la estructura de clases y cada una es afectada por la clase que la envuelve. Diferencias de clase en ideología, oportunidades de vida y estilo de vida, pueden oscurecer la naturaleza idéntica de muchos factores estructurales que afectan a las castas femeninas.

\section{Estatus y clase: problemas conceptuales y empíricos}

Planteamos un problema adicional que puede ser expresado así: si vamos a mirar a las mujeres como personas más que como apéndices de los hombres, ¿cómo definimos su estatus social, particularmente si no están trabajando por un pago y no pueden ser categorizadas en base a su propia ocupación e ingresos? ¿Se puede asignar un valor al

\footnotetext{
${ }^{8}$ Tanto Myrdal (1944, apéndice 5) como Hacker (1951) trazaron el paralelo entre las mujeres y los negros, sugiriendo que las mujeres ocupan una posición de casta similar a la de los negros. Como se señaló anteriormente, su trabajo, entre otros, ha permanecido periférico a la corriente principal de estudios de estratificación en todas las perspectivas teóricas.
} 
trabajo productivo que no es una labor pagada? ${ }^{9}$ Este es un problema más general que también emerge cuando se trata de definir el estatus de personas jubiladas, de los jóvenes que todavía son estudiantes, de los voluntarios y de los desempleados. Finalmente, también podría ser un problema incluso para determinar el estatus de hombres adultos que son parte de la fuerza de trabajo. Si se hicieran realidad las predicciones a largo plazo acerca de la menguante centralidad del trabajo y de la creciente importancia en sociedades cibernéticas de las actividades no laborales, la relevancia de la ocupación pagada en la posición de clase podría decaer, y otros elementos, como las actividades nopagadas, podrían volverse más importantes en tanto fuentes de identidad social.

Mientras tanto, una solución para superar el problema de definición del estatus social de las mujeres es ver la situación de la "ama de casa" como una ocupación y darle algún tipo de clasificación en la jerarquía de ocupaciones. A pesar de que las clasificaciones de estatus ocupacional actualmente en uso, como la escala North-Hall, no incluyen "ama de casa" como categoría, se puede desarrollar nuevas escalas. Asumo que esta ocupación se encontraría bastante abajo. Esto plantea una interesante pregunta respecto de si, y bajo qué condiciones, el matrimonio constituye un descenso de la movilidad social y/o reduce las oportunidades de movilidad para las mujeres. Al mismo tiempo, el valor de ser "ama de casa" podría variar según el estatus socioeconómico dentro del cual se da la posición. Por ejemplo, la posición de un ama de casa de clase alta podría ser mucho más valorizada en la estructura general que la posición de un ama de casa de clase baja. Podría ser que la evaluación de esta posición aumente mientras sus funciones se vuelvan más simbólicas y menos utilitarias ${ }^{10}$. O, para ponerlo de otra forma, el valor podría incrementar en cuanto sus funciones se vayan centrando más en torno al consumo y menos en torno a actividades productivas. Dentro de las clases, no obstante, la evaluación de las amas de casa respecto a otras ocupaciones disponibles para mujeres puede variar de distintas maneras.

Otra solución parcial al problema de definición del estatus de la mujer consiste en explorar más exhaustivamente el estatus conferido y del derecho de deferencia (Shils, 1968). Shils señala que "la proximidad relativa a personas en roles de poder es [otro] derecho de deferencia" (1968). Aplicado a la familia, esto significa que la posición social de la persona más poderosa en la familia es, hasta cierto punto, reflejada en los demás miembros de la familia. Las mujeres dependientes se encuentran entre las más claras receptoras de esta deferencia. Este concepto no debe ser confundido con aquel de la evaluación equivalente de todos los miembros de la familia, basada en la evaluación del hombre jefe de hogar. Aquí no hay una implicación necesaria de que sólo el jefe de hogar

\footnotetext{
${ }^{9}$ Se puede encontrar ejercicios en esta línea en revistas femeninas y en la literatura de liberación de las mujeres. Sin embargo, hasta donde sepa, no han sido seriamente seguidos por los sociólogos.

10 Thorstein Veblen, en Teoría de la clase de ocio ([1899] 1993) hace, por supuesto la misma observación.
} 
hombre determina el estatus o la posición de clase de los miembros de la familia. Algunos hombres quizás alcancen el derecho a la deferencia mediante su relación cercana con una esposa o una madre prestigiosa. Adicionalmente, estatus conferido no implica estatus equivalente. El estatus ganado mediante la asociación cercana a otra persona probablemente es de un distinto orden de deferencia de aquel que es ganado más directamente, mediante características y logros propios del individuo. Los receptores del estatus conferido, en la mayoría de los casos, probablemente no tienen deferencia equivalente con aquellos de personas cuya proximidad confiere deferencia. Con menos recursos de estatus disponibles, el receptor no puede corresponder y, por consiguiente, mientras el estatus sea conferido debe permanecer desigual para la persona con mayores recursos ${ }^{11}$. Esta relación entre el proveedor de estatus y el receptor está, muy probablemente, reflejada en evaluaciones sociales diferenciadas.

En resumen, la posición de la esposa no empleada podría ser determinada por una combinación de la clasificación de ama de casa, estatus conferido, y deferencia premarital perteneciente a la mujer en sí misma.

\section{Hacia una visión más certera de la sociedad estadounidense}

La incorporación en la sociología de la percepción de que el sexo afecta el posicionamiento en la estructura social contribuiría a una imagen más certera de nuestra sociedad. Podemos ilustrar este punto con preguntas acerca de la movilidad social y de las estructuras de poder que están involucradas cuando el sexo es tomado como una variable prominente.

Las generalizaciones acerca de los patrones de movilidad social y de las tendencias a nivel societal descansan primariamente en estudios sobre hombres blancos (Blau y Duncan, 1967). Ya que este grupo no compromete ni a la mitad de la población, la validez de estas generalizaciones puede ser cuestionada. Desde luego, la elección de los hombres como un sujeto apropiado en el estudio de la movilidad se relaciona al supuesto de que la movilidad femenina está atada a la movilidad masculina. Esto deriva de los supuestos anteriormente discutidos y también impregna la literatura. Por ejemplo, Lopata declara lo siguiente: "la clasificación ocupacional de los maridos de las mujeres entrevistadas es generalmente más alta que la de sus padres; por consiguiente las mujeres han experimentado una movilidad ascendente" $(1971,14)$.

Si desecháramos estos supuestos de la dependencia femenina, podrían emerger patrones diferentes. Por ejemplo, sería interesante mirar los patrones de movilidad intergeneracional ocupacional de las mujeres, usando el estatus de la madre como punto de origen y usando "ama de casa" como categoría ocupacional. Los hallazgos de este tipo de estudios podrían ser útiles combinados con estudios de patrones de movilidad y

\footnotetext{
${ }^{11}$ Esta idea deriva más directamente de Blau (1964).
} 
tendencias entre hombres blancos, para producir una visión mucho más compleja y completa de los procesos de movilidad social estadounidenses.

Los pocos estudios que se ha realizado acerca de la movilidad intergeneracional femenina (Rubin, 1968, 1969; Scott, 1969; Elder, 1969) se han enfocado en la movilidad mediante el matrimonio. Esto sería igualmente razonable estudiar la movilidad como una consecuencia de la disolución del matrimonio. ¿Existe, por ejemplo, una mayor probabilidad de movilidad descendente para la mujer divorciada, abandonada, o viuda que para la mujer cuyo matrimonio no ha sido interrumpido? Algunos estudios históricos sobre este problema también podrían ayudar a disolver la idea de que, incluso aunque la familia nuclear ideal no es hoy universal, fue casi-universal en un tiempo mítico de nuestro pasado. A pesar de que el divorcio masivo es un fenómeno bastante reciente en los Estados Unidos, la disolución de la familia nuclear por muerte y abandono probablemente ha estado presente siempre. La viuda digna que trabaja duro conduciendo una casa de huéspedes para pagar la educación de sus niños es una figura mítica muy conocida. Es posible que el hogar encabezado por una mujer fue más prevalente en nuestro pasado de lo que generalmente pensamos. Tal vez también puede ser que, en algunos casos, este tipo de movilidad descendente para la mujer contribuyó a los esfuerzos de movilidad en sus hijos.

Una comprensión más compleja y completa de la estructura de poder y de las relaciones de poder quizás también puede resultar del reconocimiento de la relevancia del sexo. Por ejemplo, puede existir una relación entre la posición de las mujeres y el tipo de sistema de poder. Se puede examinar este punto tanto a nivel de comunidad local como a nivel de estado-nación. Adicionalmente, comparaciones entre naciones de la posición de la mujer en sociedades que están sufriendo cambios rápidos en la estructura de clase y la distribución del poder, podrían contribuir a nuestra comprensión de sistemas sociales más amplios.

\section{Conclusión}

He indicado brevemente una serie de problemas conceptuales y empíricos que surgen si consideramos como salientes para la estructura de los sistemas de estratificación las desigualdades basadas en el sexo. A medida que la familia nuclear tradicional es cada vez menos la forma dominante en nuestro país, se volverá más visible la contribución que el sexo hace a la estructura de clase y de casta, así como al estatus social del individuo. Adicionalmente, a medida que las mujeres se vuelvan más poderosas mediante una mayor participación en la fuerza de trabajo y una organización política como mujeres, su posición en la estructura social total será un problema más legítimo para los/as sociólogos/as.

\section{BIBLIOGRAFÍA}

Bernard, Jessie (1966): Academic Women. Cleveland: World. 
(1968): The sex Game. Englewood Cliffs, N.J.: Prentice-Hall.

Blau, Peter M. (1964): Exchange and Power in Social Life. New York: Wiley.

Blau, Peter M. \& O. Dudley Duncan (1967): The American occupational structure. New York: Wiley.

Caplow, Thodore (1954): The Sociology of Work. Minneapolis: University of Minnesota Press.

Elder, Glenn H., Jr. (1969): "Appearance and Education in Marriage Mobility", en American Sociological Review 34 (Agosto): 519-32.

Ellis, Evelyn (1952): "Social Psychological Correlates of Upward Mobility among Unmarried Career Women", en American Sociological Review 17 (Octubre): 558-63.

Epstein, Cynthia (1970): "Encountering the Male Establishment: Sex Status limits on Women's Careers in the Professions", en American Journal Sociology 75 (Mayo): 965-82.

Etzioni, Amitai, ed. (1969): The Semi-Professions and Their Organizations. New York: Free Press.

Ferriss, Abbott L. (1970): Indicators of Change in the American Family. New York: Russell Sage

Haavio-Mannila, E. (1969): "Some Consequences of Women's Emancipation", en Journal of Marriage and the Family 31 (Febrero): 123-34.

Hacker, Helen Mayer (1951): “Women as a Minority Group” Social Forces 30 (Octubre) 6069.

Heller, Celia S. (1969): Structured Social Inequality. New York: Macmillan.

Hofitetter, Heather N. (1970): "The Problem of Family Status Arrangements in Stratification Analysis", Unpublished dissertation, University of Oregon.

Hughes, Everett C. (1949): "Social Change and Status Protest: An Essay on the Marginal Man", en Phylon 10 (Primer trimestre): 58-65.

Komarovsky, Mirra (1950): "Functional Analysis of Sex Roles", en American Socia1ogical Review 15 (Agosto): 508-16. .

(1953): Women in the Modem World. Boston: Little, Brown. 
Lauman, Edward O., ed. (1970): "Stratification Theory and Research", en Social inquiry 4 (Primavera): 3-12.

Lenski, Gerhard (1966): Power and Privilege. New York: McGraw-Hill.

Lopata, Helena Z. (1971): Occupation: Housewife. New York: Oxford University

Machonin, Pavel, (1970): "Social Stratification in Contemporary Czechoslovakia", en American journal of Sociology 75 (Marzo): 725-41.

Myrda1, Gunnar (1944): An American Dilemma. New York: Harper.

Oppenheimer, Valerie Kincade (1968): "The Sex-Labeling of Jobs", en Industrial Relations 7 (May): 219-34.

Rubin, Zick (1968): "Do American Women Many Up?", en American Sociological Review 33 (October): 750-60. -- (1969): "Reply to Scott", en American Sociological Review 34 (Octubre): 727-28.

Scott. J. F. (1969): "A Comment on 'Do American Women Marry Up?", en American Sociological Review 34 (October): 726-27.

Shils, Edward (1968): "Deference" in J. A. Jackson (ed.): Social Stratification. Cambridge: Cambridge University Press.

U.S. Bureau of the Census (1971): Statistical Abstract of the United States, 1971. Washington, D.C.: Government Printing Office.

Veblen, Thorstein [1899] (1953): The Theory of the Leisure Class. New York: New American Library, Mentor Books.

Watson, Walter B., \& Ernest A. Barth (1964): "Questionable Assumptions in the Theory of Social Stratification", en Pacific Sociological Review 7 (Primavera): 10-16.

Wesolowski, Wlodzimierz, and Kazimierz, Stemczynski (1968): "Social Stratification in Polish Cities", en J. A. Jackson (ed.): Social Stratification. Cambridge: Cambridge University Press.

Wilensky, Harold L. (1968): "Women's Work: Economic Growth, Ideology, Structure", en Industrial Relations 7 (Mayo): 235-48. 